\title{
Topics
}

\section{Current Status of Alloy Semiconductor Crystal Growth Project under Microgravity}

\author{
By Yuko INATOMI ${ }^{1)}$, Kaoruho SAKATA ${ }^{1)}$, Mukannan ARIVANANDHAN ${ }^{2)}$, Govindasamy RAJESH ${ }^{2)}$, Yasuhiro HAYAKAWA ${ }^{2)}$,

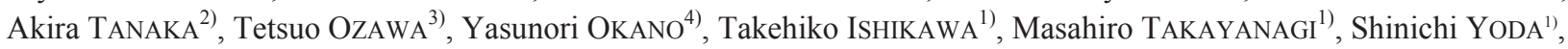 \\ and Yoshinori YoshimURA ${ }^{5)}$ \\ ${ }^{1)}$ Institute of Space and Astronautical Science, Japan Aerospace Exploration Agency, Sagamihara, Japan \\ ${ }^{2)}$ Research Institute of Electronics, Shizuoka University, Hamamatsu, Japan \\ ${ }^{3)}$ Department of Electrical Engineering, Shizuoka Institute of Science and Technology, Fukuroi, Japan \\ ${ }^{4)}$ Department of Materials Engineering Science, Osaka University, Toyonaka, Japan \\ ${ }^{5)}$ Human Space Systems Utilization Mission Directorate, Japan Aerospace Exploration Agency, Tsukuba, Japan
}

(Received June 26th, 2011)

\begin{abstract}
The purpose of "Alloy Semiconductor" crystal growth project is to make clear the factors for crystal growth of a high-quality bulk alloy semiconductor by investigating (1) solute transport in liquid and (2) surface orientation dependence of growth kinetics under microgravity and terrestrial conditions. The temperature gradient furnace Gradient Heating Furnace (GHF) onboard "Kibo" is used for the growth of an $\operatorname{In}_{x} \mathrm{Ga}_{1-x} \mathrm{Sb}$ bulk crystal which is a potential substrate material of optoelectronic devices such as thermo-photo-voltaic cells and gas sensors, since the band gap and the lattice constant of the crystals are tuned by adjusting the composition. The current status of the space experiment project will be reported in the presentation.
\end{abstract}

Key Words: Kibo, GHF, Microgravity Experiment, InGaSb, Crystal Growth

\section{Introduction}

High quality homogeneous ternary alloy semiconductor crystals are required for the fabrication of optoelectronic devices, since wavelength and lattice constant are controlled by adjusting the composition of the constituents. However, it is very difficult to grow high quality homogeneous single crystals due to the following factors: 1) segregation phenomenon, 2) temperature and concentration fluctuations caused by convection, and 3) growth kinetics at the growth interface. Since the heat and mass transport in the liquid phase are affected by buoyancy convection, the dissolution and growth processes are strongly influenced by gravity.

However, temperature and concentration gradients in the liquid become driving forces of convection in the liquid. Therefore, application of a microgravity environment is a promising method to suppress the complex convective heat and mass transports and make deeper insight into crystal growth phenomena. Several crystal growth experiments of alloy semiconductors were carried out under microgravity conditions. For example, Witt et al. grew a Te-doped InSb crystal in Skylab and achieved uniform dopant distribution ${ }^{1)}$. Kinoshita et al. grew PbSnTe crystals in the SL-J/FMPT mission, and demonstrated that the etch pit density was reduced ${ }^{2)}$. Nishinaga et al. and Duffar et al. showed that the quality of GaSb crystals was improved under microgravity ${ }^{3,4)}$.

Hayakawa et al. have performed a microgravity experiment on the dissolution and crystallization of $\operatorname{In}_{\mathrm{x}} \mathrm{Ga}_{1-\mathrm{x}} \mathrm{Sb}$ using a sandwich combination of $\mathrm{InSb}$ and $\mathrm{GaSb}$ as the starting material using the Chinese recoverable satellite ${ }^{5)}$. The effects of diffusion and Marangoni convection on the mixing of multi-component melts have been investigated in the space shuttle. It is found that the shape of the growth interface was almost flat and the Ga composition was almost uniform across the radial direction for the space-grown crystal. On the other hand, the shape of the interface broadened toward the gravitational direction and the gravitational segregation occurred on earth. These results clearly suggested that gravity affected not only the composition profiles but also the shape of the solid-liquid interface.

Therefore, it is indispensable to understand the balance among the dissolution rate of feed, growth rate of crystal, the transportation of solute in the solution, and the growth kinetics by means of in-situ observations. Inatomi et al. investigated an influence of surface orientation of a substrate crystal during solution growth process of $\mathrm{GaAs}_{\mathrm{x}} \mathrm{P}_{1-\mathrm{x}} / \mathrm{GaP}^{6}$. It was found that the growth thickness of the growth layer and the compositional distribution in the layer obviously depend on the surface orientation of the substrate even using a solvent with a same initial composition during the growth. However, when compared to binary compounds, the dissolution process and growth kinetics are more complicated for ternary compounds. Rajesh et al. have investigated the dissolution process of GaSb into InSb melt during growth of $\mathrm{In}_{\mathrm{x}} \mathrm{Ga}_{1-\mathrm{x}} \mathrm{Sb}$ in situ by an X-ray penetration method. The experimental indicated that the shape of the growth interface and composition profiles were strongly influenced by buoyancy convection ${ }^{7)}$. 
The purpose of "Alloy Semiconductor" crystal growth project is to make clear the factors for crystal growth of a high-quality bulk alloy semiconductor by investigating (1) solute transport in liquid and (2) surface orientation dependence of growth kinetics under microgravity and terrestrial conditions. If the homogeneous and high-quality bulk crystal with desired composition can be grown based on the results of the space experiment, the knowledge to grow suitable thin layers on the crystal will be achieved for fabrication of the optoelectronic devices. This experiment was selected as one of the 14 candidate mission of "Kibo" Second Phase Utilization in 2008. The Japanese Experiment Module "Kibo" is Japan's first manned experiment facility on the International Space Station. The temperature gradient furnace Gradient Heating Furnace (GHF) onboard Kibo is used for the growth of an $\mathrm{In}_{\mathrm{x}} \mathrm{Ga}_{1-\mathrm{x}} \mathrm{Sb}$ bulk crystal, whose melting point is lower than $712^{\circ} \mathrm{C}$. Since no in situ observation facility for semiconductor crystal growth has been installed in ISS at this point, a thermal-pulse technique is periodically applied to make impurity striations in a grown crystal as time markers artificially. The present paper describes the current status of the space experiment project.

\section{Experimental}

\subsection{Crystal growth facility in space}

The Japanese Experiment Module "Kibo" is a Japanese science module for the International Space Station. Kobairo Rack, which contained GHF, was installed into "Kibo" in 2011. Figure 1 shows a GHF system configuration. GHF is a vacuum furnace with automatic sample exchange mechanism that can be accommodate up to 15 samples. Their positions and temperatures of the three heating zones can be independently controlled. The heating zones can be independently moved along one axis. GHF is operated automatically in accordance with the temperature profile of each heater. The progress of their experiment can be confirmed with temperature data downlinked from Kibo.
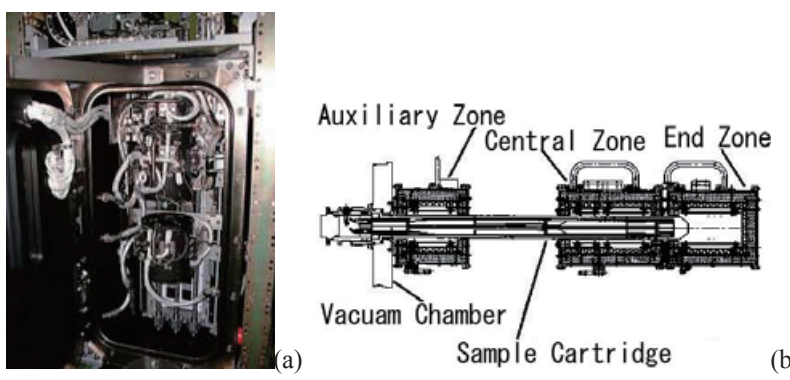

Fig. 1. Gradient Heating Furnace: (a) photo of flight model, (b) schematics of heating zone with sample cartridge.

\subsection{Experimental procedure}

$\mathrm{In}_{\mathrm{x}} \mathrm{Ga}_{1-\mathrm{x}} \mathrm{Sb}$ bulk crystals are grown on a GaSb seed under a constant temperature gradient using five $\mathrm{GaSb}$ (seed)/InSb/GaSb(feed) sandwich samples. The list of the sample ampoule for the space experiment is shown in Table 1. Tellurium is doped in the InSb crystal. A GaSb feed is dissolved into InGaSb solution to supply GaSb component during the growth. In order to measure the growth rate, tellurium impurity striations are intentionally introduced into the crystal by thermal-pulse technique.

The outer size of the sample ampoule is $13 \mathrm{~mm} \varphi \times 95 \mathrm{~mm}$ long. The cylindrical shaped $\mathrm{GaSb}$ (seed)/InSb/GaSb(feed) sandwich sample is inserted in a BN tube and then put into a quartz ampoule. The upper $\mathrm{GaSb}$ feed is set up at high temperature region and lower $\mathrm{GaSb}$ seed is set up at low temperature region. The sizes of the crystals are the same as those adopted in the Chinese recoverable satellite experiment. To prevent accidental breaking of the ampoule and to adjust the volume change from solid to liquid and vice versa, both the ends of the sample are packed by carbon sheets. The ampoule is evacuated to $10^{-4} \mathrm{~Pa}$, and then sealed off. The rough design of the ampoule is shown in Fig. 2. Temperature profile in the sample is monitored by thermocouples place on the surface of the outer cartridge described below. Since the sample is covered by the BN tube and quartz ampoule, the sample temperature would be reasonably varied from the temperature on the cartridge surface.

Table 1. List of sample ampoule for space experiment.
\begin{tabular}{|c|c|c|c|}
\hline No. & Purpose & Seed crystal & Feed crystal \\
\hline 1 & Temperature calibration & $\mathrm{GaSb}(111) \mathrm{A}$ & $\mathrm{GaSb}(111) \mathrm{A}$ \\
\hline 2 & $\mathrm{GaSb}(111)$ A growth & $\mathrm{GaSb}(111) \mathrm{A}$ & $\mathrm{GaSb}(111) \mathrm{A}$ \\
\hline 3 & $\mathrm{GaSb}(111) \mathrm{B}$ growth & $\mathrm{GaSb}(111) \mathrm{B}$ & $\mathrm{GaSb}(111) \mathrm{B}$ \\
\hline 4 & $\mathrm{GaSb}(100)$ growth & $\mathrm{GaSb}(100)$ & $\mathrm{GaSb}(100)$ \\
\hline 5 & $\mathrm{GaSb}(110)$ growth & $\mathrm{GaSb}(110)$ & $\mathrm{GaSb}(110)$ \\
\hline
\end{tabular}

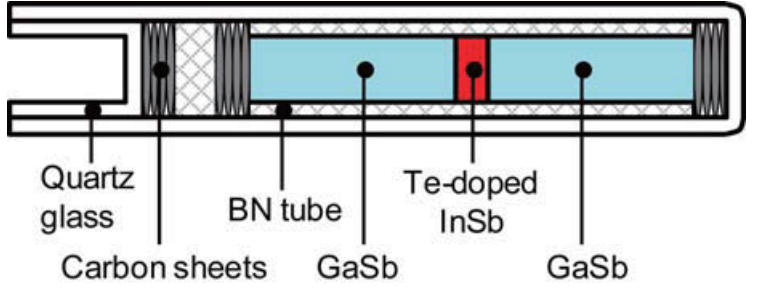

Fig. 2. Schematic of sample ampoule for crystal growth.

The crystal growth procedure is as follows. (1) The cartridge surface is heated up to $700^{\circ} \mathrm{C}$ at a heating rate of $30^{\circ} \mathrm{C} / \mathrm{h}$. Subsequently position and temperature of each heater zones are fixed for $82 \mathrm{~h}$. (2) In the initial stage of the heating, the InSb crystal is completely melted and the GaSb seed is partially melted. The GaSb feed dissolves into the InSb melt and the InGaSb solution is formed. (3) The temperature at the solution/feed interface is higher than that at the seed/solution interface, which causes the GaSb composition at the solution/feed interface to be higher than that of seed/solution interface. Therefore, the GaSb moves to the seed/solution interface by diffusion due to GaSb composition gradient between the solution/feed and seed/solution interface. (4) The solution near the seed/solution interface becomes supersaturated, which cause spontaneous $\mathrm{In}_{\mathrm{x}} \mathrm{Ga}_{1-\mathrm{x}} \mathrm{Sb}$ crystal growth at the seed/solution interface without moving the heater zones and without decreasing temperature of the sample. The seed/solution interface slowly moves towards the solution/feed interface due to spontaneous crystal growth. The 
temperature gradient and the maximum temperature in the crystals are required to be about $6^{\circ} \mathrm{C} / \mathrm{cm}$ and lower than $710^{\circ} \mathrm{C}$, respectively.

\section{Preparation for Space Experiment}

\subsection{Design of sample cartridge}

The sample cartridge consists of the following three parts: Sample, Support and Boss. In the Sample part the ampoule is vacuum-encapsulated by a cylindrical cartridge made of C-103 (melting point: $2350^{\circ} \mathrm{C}$ ), which is considered as the most suitable Niobium alloy from the standpoint of welding and the high-temperature strength. Sample part is connected to Boss part with Support part made of C-103 rigidly during heating in GHF. Boss part has a roll of mechanical, thermal and electrical interfaces between the cartridge and GHF. The tips of four Tungsten- Rhenium thermocouples are place on the surface of Sample part.

In order to evaluate safety of the cartridge material in GHF experiment, evaporation rate of $\mathrm{InGaSb}$ melt and wettability of C-103 with the melt were measured as a function of temperature. The evaporation rates of $\mathrm{GaSb}, \mathrm{InSb}$ and $\mathrm{In}_{0.4} \mathrm{Ga}_{0.6} \mathrm{Sb}$ melts at $1105^{\circ} \mathrm{C}$ were less than $5 \times 10^{-5} \mathrm{~g} / \mathrm{cm}^{2} \mathrm{sec}$, which was a negligible value from the viewpoint of safety in Kibo. Figure 3 shows the temperature dependence of wettability of quartz glass, boron nitride, graphite, and C-103 with $\mathrm{In}_{0.4} \mathrm{Ga}_{0.6} \mathrm{Sb}$ melt. The initial shape of the InGaSb crystal was cubic. The surface of the $\mathrm{C}-103$ substrate was covered with the melt well at $1100^{\circ} \mathrm{C}$, while the sphere melt kept the shape on the other surface at the temperature. Thus, the wettability of $\mathrm{C}-103$ is preferable to prevent penetration of the cartridge wall at small area by InGaSb melt during the heating, even if the melt leaks from cracks of the quartz glass ampoule due to an accident. Consequently the minimum thickness of the cartridge wall was determined as $4 \mathrm{~mm}$ from the result of a corrosion test with the melt at $1105^{\circ} \mathrm{C}$.

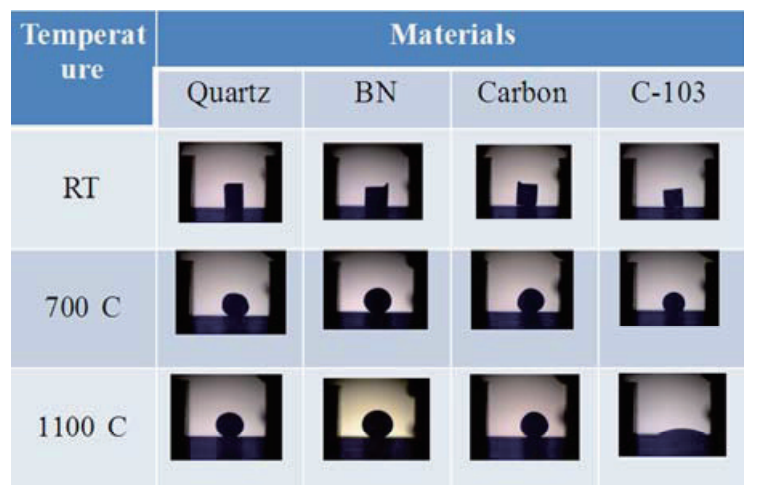

Fig. 3. Wettability of quartz, boron nitride, carbon and C-103 substrates with InGaSb solution as a function of temperature.

Numerical simulations for heat transfer characteristic in GHF were performed using the commercial software package FIDAP 8.7.4, which was based on the finite element method. All calculations were carried out with a non-uniform four-node quadrilateral element mesh for the sample cartridge, the heater elements, and the outer case. The detailed shape of the sample cartridge, the position of the heater elements and the sample cartridge, and the temperature program of each heater were optimized by the simulation. Figure 4 shows an example of the temperature distribution in GHF calculated by the simulations. The cartridge surface was roughened by sandblasting in order to increase the emissivity of the surface over 0.9 , because it was found by the simulation that thermal response of the sample was improved by an increase in the emissivity. The emissivity of the sample surface at high temperature was checked by an Fourier transform infrared spectroscopy measurement measurement of the sample.

$\mathrm{GaSb}$ and $\mathrm{InSb}$ single crystals for the space experiment were grown by the Czochralski method. These crystals were preferably formed into a cylindrical shape. Figure 5 shows the engineering model fabricated on the basis of the simulation results and upon request of the safety.

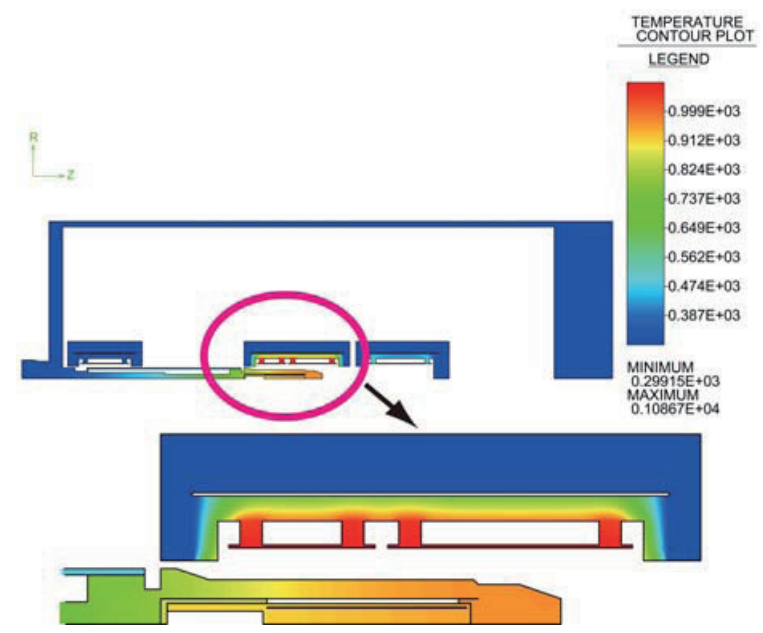

Fig. 4. Calculated temperature distribution in GHF.

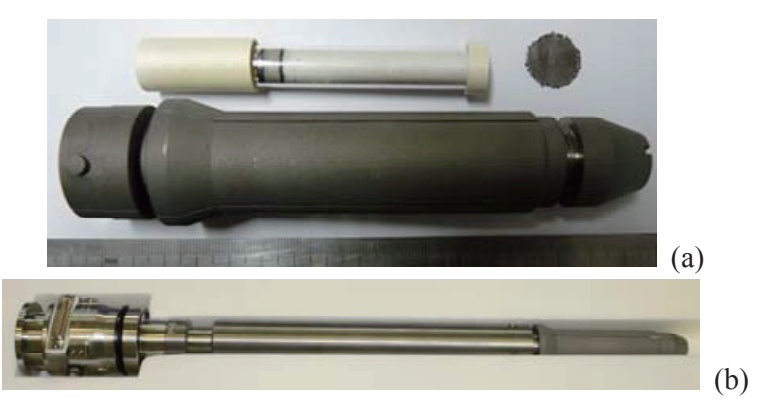

Fig. 5. Engineering model of sample cartridge: (a) Sample part and (b) fabricated cartridge for environmental test.

\subsection{Environmental test}

The sample cartridges for Alloy Semiconductor experiment will be delivered to the ISS on the HTV3 Mission in 2011. The cartridges after the experiments in orbit are planned to be returned to the ground by a reusable spacecraft. Therefore, environmental test for simulating vibration and shock during the launch and the recovery has been performed to evaluate the optimization of the cartridge structure. There was a possibility that the InSb crystal in the ampoule was cracked by mechanical vibration or shock, because the crystal is more 
ductile than GaSb crystal. As a countermeasure against the cracking of the InSb crystal, amount of the carbon sheets was adjusted to push the crystals in the ampoule moderately. The soundness of the structure in the ampoule was confirmed by an X-ray transmission method, because boron nitride and quartz glass were transparent materials for X-ray. Consequently it was made sure that the sample cartridge could be used safely for the microgravity experiment.

\subsection{Growth rate and concentration in crystal}

The $\mathrm{In}_{\mathrm{x}} \mathrm{Ga}_{1-\mathrm{x}} \mathrm{Sb}$ crystals were grown by the temperature gradient method on earth with the same configuration as the flight model. During the crystal growth, thermal pulses were introduced to yield dopant striations in the grown crystal by changing the heater temperature as a delta-function of time. The growth rate can be obtained from the ratio of the striation distance divided by the period of the thermal pulse. It is necessary to adjust height and width of the temperature change in order to make the contrast of the striations to be sharp. The striations in the grown crystal were observed by chemical etching as shown in Fig. 6. The compositions of the grown crystals were determined by means of Electron Probe Micro-Analysis (EPMA). Figure 7 shows the measurement result of concentration distribution of $\mathrm{In}, \mathrm{Ga}$ and $\mathrm{Sb}$ in the grown crystal by EPMA. The local temperature gradient in the sample can be estimated from the indium composition profile in the grown crystal. The indium composition profile is converted to the growth temperature profile using a solidus line of the InSb-GaSb pseudo-binary phase diagram ${ }^{8)}$.

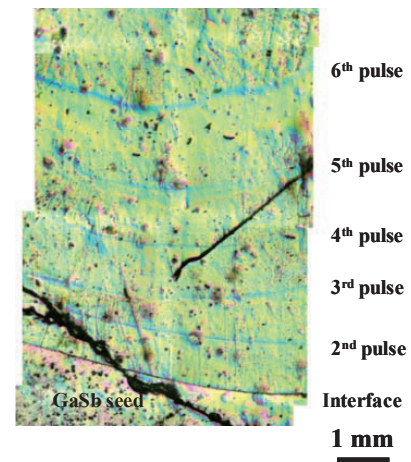

Fig. 6. Impurity striations in grown crystal as time markers. Narrow blue lines correspond to the striations.
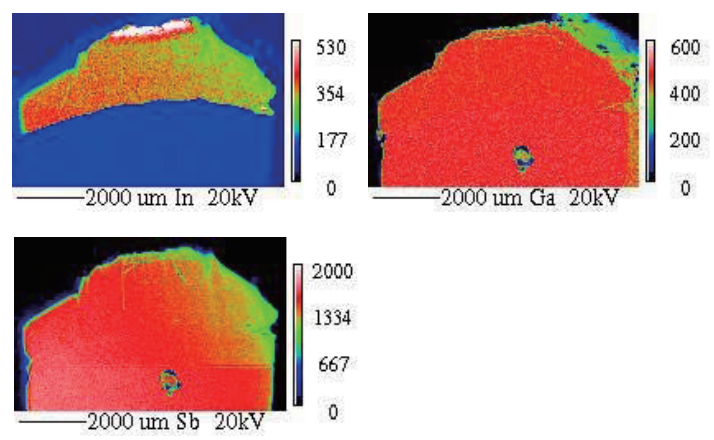

Fig. 7. Concentration distribution of $\mathrm{In}, \mathrm{Ga}$ and $\mathrm{Sb}$ in grown crystal.

\section{Conclusion}

$\mathrm{In}_{\mathrm{x}} \mathrm{Ga}_{1-\mathrm{x}} \mathrm{Sb}$ crystals are grown by a temperature gradient method by utilizing a microgravity environment in Kibo, and the morphological change of the growth interface is visualized by a thermal-pulse technique. The sample cartridge was designed based on the result of the numerical simulation and upon request of the safety by taking account of usage of GHF. It was made sure that the sample cartridge could be used safely for the microgravity experiment by environmental tests.

\section{Acknowledgments}

The part of the work was supported by the cooperative research projects of the Research Institute of Electronics, Shizuoka University. This work was also financially supported by a Grant-in-Aid for Scientific Research (B) (no.22360316) and a Grant-in-Aid for Young scientist B (no. 22760005) from the Ministry of Education, Culture, Sports, Science and Technology of Japan.

\section{References}

1) Witt, A. F., Gatos, H. C., Lichtensteiger, M., Lavine, M. C. and Herman, C. J.: Crystal Growth and Steady-State Segregation under Zero Gravity: InSb, J. Electrochem. Soc. 122 (1978), pp. 276-283.

2) Kinosita, K. and Yamada, T.: $\mathrm{Pb}_{1-\mathrm{x}} \mathrm{Sn}_{\mathrm{x}} \mathrm{Te}$ Crystal Growth in Space, J. Crystal Growth 147 (1995), pp. 91-98.

3) Nishinaga, T., Ge, P., Huo, C., He, J. and Nakamura, T.: Melt Growth of Striation and Etch Pit Free GaSb under Microgravity, $J$. Crystal Growth 174 (1997), pp. 96-100.

4) Duffar, T., Serrano, M. D., Moore, C. D., Camassel, J., Contreras, S., Dusserre, P., Rivoallant, A. and Tanner, B. K.: Bridgman Solidification of GaSb in Space, J. Crystal Growth 192 (1998), pp. 63-72.

5) Hayakawa, Y., Balakrishnan, K., Arafune, K., Ozawa, T., Okano, Y., Hirata, A. and Kumagawa, M.: "Microgravity Experiments on Dissolution and Crystallization of InGaSb" in Studies on Crystal Growth under Microgravity, edited by Y. Hayakawa and Y. Furukawa: Transworld Research Network (2005), pp. 1-50.

6) Inatomi, Y., Kikuchi, M., Nakamura, R., Kuribayashi, K. and Jimbo, I.: In situ observation for semiconductor solution growth using a near-infrared microscope, J. Crystal Growth 275 (2005), pp. 193-200.

7) Rajesh, G., Arivanandhan, M., Morii, H., Aoki, T., Koyama, T., Momose, Y., Tanaka, A., Ozawa, T., Inatomi, Y. and Hayakawa, Y.: In-situ observations of dissolution process of GaSb into InSb melt by X-ray penetration method, J. Crystal Growth 312 (2010), pp. 2677-2682.

8) Stringfellow, G. B.: Calculation of Ternary Phase Diagrams of III-V Systems, Phys. Chem. Solids 33 (1972), pp. 665-677. 